\title{
Abordagem da temática aproveitamento total dos alimentos no Ensino de Ciências da Natureza com enfoque interdisciplinar: relato de experiência no contexto da Educação Básica
}

Approach to the theme of total use of food in the Teaching of Natural Sciences with an interdisciplinary approach: experience report in the context of Basic Education

\section{Aproximación al tema del uso total de los alimentos en la Enseñanza de las Ciencias Naturales con un enfoque interdisciplinario: relato de experiencia en el contexto de la Educación Básica}

\author{
Francielle Escobar Esteve (francielleescobar25@gmail.com) \\ Universidade Federal do Pampa-Unipampa.
}

Franciele Braz de Oliveira Coelho (francielecoelho@unipampa.edu.br)

Universidade Federal do Pampa-Unipampa.

Resumo: Apresentamos um relato de experiência tendo como foco o Ensino de Ciências da Natureza através da temática aproveitamento total dos alimentos, por meio de atividades com viés interdisciplinar. Participaram das atividades, estudantes de primeira série do Ensino Médio de uma escola pública estadual da região da Campanha do Estado do RS. As atividades desenvolvidas estavam vinculadas ao Estágio Supervisionado Obrigatório de um curso de Licenciatura em Ciências da Natureza. No desenvolvimento foi aplicada uma oficina temática com foco na interdisciplinaridade, por meio da metodologia de Delizoicov e Angotti (1994), denominada de Três Momentos Pedagógicos (TMP). As intervenções demonstraram como principais resultados: conscientização sobre ações sustentáveis relacionadas à alimentação e a qualidade do ambiente em que vivemos; compreensão de conceitos de Ciências da Natureza a partir de situações do cotidiano; crescente motivação dos estudantes quando ocorre a diversificação de recursos didáticos para o aprendizado de Ciências; destaque para a utilização de oficina temática aliada aos TMP na promoção do conhecimento. Dessa forma, obtiveram-se resultados positivos, principalmente em relação à importância da inovação nas metodologias de ensino na Educação Básica.

Palavras-chave: Interdisciplinaridade; Ensino de Ciências; Alimentação; Ensino Médio.

Abstract: We present an experience report focusing on the Teaching of Natural Sciences through the theme of total use of food, through activities with an interdisciplinary bias. Participated in the activities, students of first grade of High 
School of a public state school of the region of the Campaign of the State of RS. The activities developed were linked to the Mandatory Supervised Internship of a degree course in Natural Sciences. In the development, thematic workshops were applied with a focus on interdisciplinarity, using the methodology of Delizoicov and Angotti (1994), called Three Pedagogical Moments (TMP). The interventions showed as main results: awareness of sustainable actions related to food and the quality of the environment in which we live; understanding of concepts of Natural Sciences from everyday situations; growing motivation of students when there is a diversification of didactic resources for science learning; emphasis on the use of a thematic workshop combined with TMP in promoting knowledge. In this way, positive results were obtained, mainly in relation to the importance of innovation in teaching methodologies in Basic Education.

Keywords: Interdisciplinarity; Science teaching; Food; High school.

Resumen: Presentamos un relato de experiencia centrado en la Enseñanza de las Ciencias Naturales a través del tema del uso total de los alimentos, a través de actividades con un sesgo interdisciplinario. Participaron de las actividades, alumnos de primer grado de Bachillerato de un colegio público estatal de la región de la Campaña del Estado de RS. Las actividades desarrolladas estuvieron vinculadas a la Pasantía Obligatoria Supervisada de una carrera de Licenciatura en Ciencias Naturales. En el desarrollo se aplicaron talleres temáticos con enfoque de interdisciplinariedad, utilizando la metodología de Delizoicov y Angotti (1994), denominada Tres Momentos Pedagógicos (TMP). Las intervenciones mostraron como principales resultados: sensibilización sobre acciones sostenibles relacionadas con la alimentación y la calidad del medio ambiente en el que vivimos; comprensión de conceptos de Ciencias Naturales a partir de situaciones cotidianas; creciente motivación de los estudiantes cuando existe una diversificación de recursos didácticos para el aprendizaje de las Ciencias; énfasis en el uso de un taller temático combinado con TMP en la promoción del conocimiento, de esta manera se obtuvieron resultados positivos, principalmente en relación a la importancia de la innovación en las metodologías de enseñanza en Educación Básica.

Palabras llave: Interdisciplinariedad; Enseñanza de las ciencias; Alimentación; Escuela secundaria.

\section{INTRODUÇÃO}

Frequentemente ocorre o desperdício de alimentos por preconceito, mito, falta de hábito, desconhecimento etc. Apesar disso, existem vários motivos pelos quais as pessoas se beneficiarão ao utilizar melhor os alimentos, incluindo partes que convencionalmente seriam descartadas. Segundo a Organização das Nações Unidas para Alimentação e Agricultura $(\mathrm{FAO})^{1}$ toneladas de alimentos são desperdiçadas

${ }^{1}$ Relatório da FAO (Organização das Nações Unidas para Alimentação e Agricultura) Disponível em http://nacoesunidas.org/desperdicio-de-alimentos-custa-ao-mundo-750-bilhoes-de-dolares-alerta-novorelatorio-da-fao/. Acesso em 29 ago 2019. 
anualmente, cerca de 1,3 bilhões de toneladas em todo o mundo, causando grandes perdas econômicas e também um impacto nos recursos naturais que são de extrema importância para a humanidade. A falta de conhecimento da população sobre as propriedades nutricionais dos alimentos é um dos fatores que levam ao desperdício.

Verificando o histórico das políticas sociais de nosso país que buscaram relacionar produção, abastecimento e consumo de alimentos aliados a nutrição, o setor da saúde foi o principal protagonista na proposição de respostas aos problemas da insegurança alimentar e nutricional da população (VASCONCELOS, 2005; PINHEIRO, 2008). Tal fato é observado "[...] porque é nos serviços de saúde que recaem os problemas decorrentes da fome, expressa pela desnutrição, e de um sistema alimentar não promotor da saúde que gera doenças relacionadas às práticas alimentares inadequadas, tal como a obesidade" (JAIME et al, 2018, p. 1834). Baumgratz et al (2019) enfatizam que os estudantes realizam no mínimo uma refeição ao dia no ambiente escolar, o que possibilita que a reeducação alimentar seja introduzida neste espaço. A temática também pode ser contemplada no Ensino de Ciências da Natureza, buscando promover a construção do conhecimento articulado com o cotidiano dos estudantes. Partes de alimentos e sobras podem ser aproveitadas, tornando-se receitas novas e deliciosas, o que permite em sala de aula a exploração de conceitos de Ciências envolvendo a temática.

Neste trabalho, relatamos uma experiência vivenciada em uma turma de Ensino Médio, com a aplicação de uma oficina temática, visando estabelecer relações entre o aproveitamento total dos alimentos e a interdisciplinaridade. O desenvolvimento de um trabalho interdisciplinar no contexto educacional pode colaborar com o processo de construção de conhecimento e promover no educando o senso crítico e participativo, além de tornar as aulas mais dinâmicas e interessantes. Para tanto, se faz necessário “[...] ensinar os métodos que permitam estabelecer as relações mútuas e as influências recíprocas entre as partes e o todo em um mundo complexo" (MORIN, 2000, p. 14).

Com o desenvolvimento da oficina, objetivou-se analisar a contribuição desta no processo de construção do conhecimento dos estudantes da Educação Básica, para a compreensão de conceitos de Ciências da Natureza relacionados ao aproveitamento total de alimentos. Na sequência, apresentamos o referencial teórico que embasou o 
desenvolvimento das intervenções, a metodologia adotada, os principais resultados obtidos e nossas considerações finais.

\section{ALIMENTOS COMO TEMÁtICA PARA O ENSINO INTERDISCIPLINAR DE CIÊNCIAS DA NATUREZA}

As relações estabelecidas no contexto de sala de aula são redefinidas quando há a adoção de uma postura interdisciplinar pelo professor. Espera-se, atualmente, que o docente seja "[...]capaz de enfrentar um novo desafio ao ter que iniciar uma nova disciplina ou uma nova estratégia pedagógica inspirada nos princípios da complexidade, da interdisciplinaridade e da transdisciplinaridade" (MORAES, 2007, p. 19).

A interdisciplinaridade não surge como proposta de se criar diferentes disciplinas, mas atua com o propósito de compreender os conhecimentos de várias disciplinas, auxiliando o trabalho de temas antes trabalhados isoladamente (BONATTO et al, 2012). A conexão entre as disciplinas que a interdisciplinaridade propõe, pode contribuir no Ensino de Ciências da Natureza, pois permitirá desenvolver reflexões em torno do exercício da cidadania. Quando o ensino parte de uma problematização, a interdisciplinaridade auxilia na compreensão e exploração dos conceitos, uma vez que a disciplina isolada não possibilita a mesma visão. Feistel (2012) ressalta aspectos da concepção freireana de Educação que favoreceriam o desenvolvimento de um trabalho com viés interdisciplinar em sala de aula: trabalho coletivo, diálogo entre os pares, reconhecimento do processo histórico, filosófico e sociológico do conhecimento.

Assim,

[...] para que a interdisciplinaridade chegue ao cotidiano da escola, é necessário que os professores se sintam em condições de inseri-la em seu contexto de ensino. Para isso, uma possibilidade é criar espaços de diálogo para que os professores de diferentes áreas possam trocar experiências e elaborar ações conjuntas (LOCATELLI; CRESTANI; ROSA, 2020, p. 190).

A temática de aproveitamento total dos alimentos permite o desenvolvimento de ações interdisciplinares, que promovam o Ensino de Ciências da Natureza nas escolas. Com relação à Educação Alimentar, os autores Amaral e Almeida (2015) também reforçam que o tema pode ser tratado de forma interdisciplinar, abordando diferentes aspectos e assuntos por diversas áreas do saber. O tema alimentação pode 
contribuir com o Ensino de Ciências da Natureza, com o desenvolvimento de atividades que integrem educação alimentar e saúde, ampliando os conhecimentos dos estudantes e, proporcionando uma aproximação entre as diferentes áreas do saber, em que o ensino ocorra de forma interdisciplinar.

\subsection{OFICINAS TEMÁTICAS E O ENSINO DE CIÊNCIAS DA NATUREZA}

As oficinas temáticas proporcionam por meio da busca de soluções para um determinado problema, a partir de conhecimentos do cotidiano do estudante, uma proposta para os processos de ensino e de aprendizagem (MARCONDES, 2008). Para Marcondes (2006) “[...] uma oficina temática é um trabalho fundamentado no princípio da contextualização" (p. 23). A contextualização é citada nas Diretrizes Curriculares Nacionais do Ensino Médio (DCNEM) (BRASIL, 1998), sendo destacado que: “[...] a aplicação de conhecimentos constituídos na escola às situações da vida cotidiana e da experiência espontânea permite seu entendimento, crítica e revisão" (p. 04).

Aliada à contextualização e o uso de experimentação, oficinas temáticas podem colaborar significativamente para o processo de construção de conhecimento dos estudantes em relação aos conceitos de Ciências da Natureza. As oficinas temáticas permitem a aproximação entre o conhecimento científico e o conhecimento escolar, favorecendo o entendimento de conceitos abstratos e complexos da Ciência.

\section{METODOLOGIA}

Para o trabalho apresentado neste relato de experiência, na abordagem do tema aproveitamento total dos alimentos foi dinamizada uma oficina temática, desenvolvida em quatro etapas com foco na interdisciplinaridade para estudantes de Ensino Médio de uma escola da rede pública estadual do RS. A oficina seguiu a metodologia de Delizoicov e Angotti (1994) denominada Três Momentos Pedagógicos (TMP), que organizam o processo de construção do conhecimento em três etapas, sendo elas: problematização inicial; organização do conhecimento e aplicação do conhecimento. Em que, segundo os autores:

Num primeiro momento o aluno está com a palavra; ou seja, o professor ouve o que o aluno tem a dizer sobre o assunto: tanto sua maneira de entender o conteúdo, como também a sua experiência de vida. Um segundo momento no qual, a partir da colocação dos alunos através de atividades, o professor ensina um conteúdo novo à classe. Um terceiro momento, no qual o aluno é 
estimulado a aplicar este conhecimento a uma situação nova, ou a explicá-lo com suas próprias palavras, ou elaborar um trabalho qualquer, retrabalhando o que aprendeu, apropriando-se do conhecimento adquirido (DELIZOICOV; ANGOTTI, 1994, p. 128).

Portanto, utilizando a metodologia de ensino dos TMP, pode-se levar o estudante a uma compreensão mais significativa, valorizando seu conhecimento prévio, sendo indispensável também a participação do professor, que se torna um mediador no processo de construção do conhecimento. No desenvolvimento da oficina temática descrita nesse relato, foram abordados alguns conceitos de Ciências da Natureza atrelados ao tema aproveitamento total dos alimentos. Dentre os conceitos desenvolvidos, foram abordados: energia mecânica, energia química, potência mecânica, propagação da energia térmica, fermentação química e componentes nutricionais dos alimentos. Na sequência, são descritas as atividades desenvolvidas na oficina.

\subsection{OFICINA TEMÁTICA SOBRE APROVEITAMENTO TOTAL DOS}

\section{ALIMENTOS}

Na primeira etapa da oficina foi desenvolvido o momento da problematização inicial com duração de $1 \mathrm{~h}$, em que, foram identificados os conhecimentos prévios dos participantes, por meio de uma prática investigativa, com as questões descritas na Tabela 01:

\section{Questões sobre a temática aproveitamento total dos alimentos}

\footnotetext{
A) Você tem o hábito de aproveitar os alimentos de forma integral? De que forma?

B) Você já ouviu o termo "sustentabilidade"?

C) O que você sabe sobre o aproveitamento de alimentos em sua totalidade?
}

\section{Tabela 01 - Questões da prática investigativa.}

Na segunda etapa da oficina foi desenvolvido o segundo momento pedagógico, a organização do conhecimento, com duração de 1 h. Nesta etapa os estudantes construíram uma roda de alimentos, sendo esta uma representação gráfica em forma de círculo que ajuda a combinar os alimentos que deverão fazer parte da alimentação diária. Ela está dividida em sete grupos de alimentos: cereais e derivados e tubérculos (28\%), hortícolas (23\%), fruta (20\%), laticínios (18\%), carne, peixe e ovos (5\%), leguminosas (4\%), gorduras e óleos (2\%) (RODRIGUES et al, 2006). A roda de alimentos indica a proporção correta de cada alimento, baseado em uma alimentação 
saudável. A água estará no centro da roda, pois está presente na constituição de todos os alimentos, tornando-se vital para nossa sobrevivência.

$\mathrm{Na}$ terceira etapa da oficina, foi utilizado recurso áudio visual, e neste momento os estudantes assistiram a um vídeo ${ }^{2}$ que abordava a temática. O vídeo aborda os componentes nutricionais dos alimentos e a importância dos mesmos em nosso organismo. Logo após, iniciou-se o processo de fabricação de uma receita com aproveitamento de cascas de maçã. Para produção do cupcake foi adotada a seguinte receita:

\section{Receita: Cupcake de Casca de Maçã}

Ingredientes

1 xícara de farinha de trigo; 1 xícara de açúcar; $1 / 2$ xícara de manteiga derretida; 2 maçãs; 2 colheres de canela em pó; 1 colher de fermento químico em pó; 1 ovo; Forminhas de papel para cupcakes; Forminhas de alumínio ou forma de cupcakes.

\section{Modo de preparo}

- Lavar e higienizar as maçãs com casca em solução clorada.

- Descascar as maçãs e reservar as cascas.

- No liquidificador, colocar a manteiga derretida, o ovo, as cascas das maçãs e o açúcar. Bater tudo.

- Picar as maçãs em cubos.

- Numa bacia, acrescentar a mistura do liquidificador e as maçãs em cubos. Aos poucos ir acrescentando a farinha de trigo, por último acrescentar o fermento em pó, mexendo até misturar bem.

- Colocar as forminhas de papel posicionadas sobre as formas de alumínio e preencher com a massa até a metade da forminha. Misturar a canela e o açúcar e polvilhar por cima da massa nas forminhas. Levar ao forno preaquecido por 25 minutos a $180^{\circ} \mathrm{C}$.

RV/IST2 DSIOV Tabela 02- Receita de cupcake com casca de maçã.

Fonte: G1- Globo.com

Durante o processo de produção do bolo, foram explorados conceitos de Ciências da Natureza: alimentação; energia e potência; propagação de energia térmica, componentes nutricionais dos alimentos, que foram introduzidos através de questões como:

A) Que tipos de nutrientes estão presentes em maior quantidade em cada produto utilizado? (Alimentação e componentes nutricionais)

B) Com base nas quantidades de cada ingrediente acrescentado na receita do bolo, determine sua energia, em Kcal? Qual potência, em watts, é produzida por esta energia se ingerida por um adulto? (Energia e potência)

\footnotetext{
${ }^{2}$ Disponível em: <https://www.youtube.com/watch?v=PtfyEYGQsf0>. Acesso 20 nov 2019.
} 
C) Durante o preparo do bolo, quais os processos de propagação de energia térmica envolvidos em cada etapa? Conceitue cada processo. (Propagação de energia térmica)

Ao final as receitas foram degustadas, e através do diálogo estimulou-se novamente, a importância de utilizar melhor os recursos naturais. Esta etapa da oficina foi realizada com a distribuição das receitas impressas aos participantes. Na última etapa da oficina, os estudantes com o auxílio de um software, representaram em um infográfico o que tinham estudado durante as etapas desenvolvidas na oficina. Essa atividade foi realizada em duplas. Após o desenvolvimento desta etapa, apresentou-se os infográficos com a socialização dos resultados. Os resultados obtidos são descritos a seguir.

\section{RESULTADOS E DISCUSSÕES}

Buscando verificar as concepções prévias dos participantes sobre a temática aproveitamento integral dos alimentos e conceitos de Ciências da Natureza relacionados ao tema, os estudantes responderam à um questionário. Para Vygostsky “[...] qualquer situação de aprendizado tem sempre uma história prévia, sendo necessária a abordagem do conhecimento que eles já possuem" (2000, p. 210). Como principais resultados desta etapa da oficina, foi possível identificar que: (a) a maioria dos estudantes não apresentava hábitos alimentares saudáveis; (b) os estudantes julgaram que a melhor alternativa para o descarte do lixo, seria a associação das ações: coleta seletiva e reciclagem; (c) a maioria dos estudantes da turma não tinha conhecimento sobre a alimentação sustentável. Esta pode ser considerada como uma atitude que visa equilibrar os recursos naturais que existem, causando menos danos ao meio ambiente, tais atitudes incluem aproveitar melhor os alimentos e escolher frutas, verduras e legumes adequados a cada época do ano; (d) os estudantes acreditavam que os carboidratos eram os principais responsáveis pela manutenção de funções importantes do corpo humano, seguido dos nutrientes e da energia. Sobre a ingestão de carboidratos, vale ressaltar que estes: “[...] possuem função principalmente energética. São utilizados pelos músculos para realização de movimentos e são armazenados no fígado para manutenção da glicemia" (BRASIL, 2002, p. 15). 
Partindo das concepções prévias da turma, foram dinamizadas as demais etapas da oficina, descritas na metodologia deste relato de experiência. A realização do segundo momento pedagógico, denominado organização do conhecimento, ocorreu nas etapas 02 e 03 da oficina. A segunda etapa, teve como objetivo a construção de uma representação gráfica da roda de alimentos. Durante o desenvolvimento desta atividade os participantes se mantiveram envolvidos e bastante participativos. A construção da roda de alimentos pode ser verificada na Imagem 01:

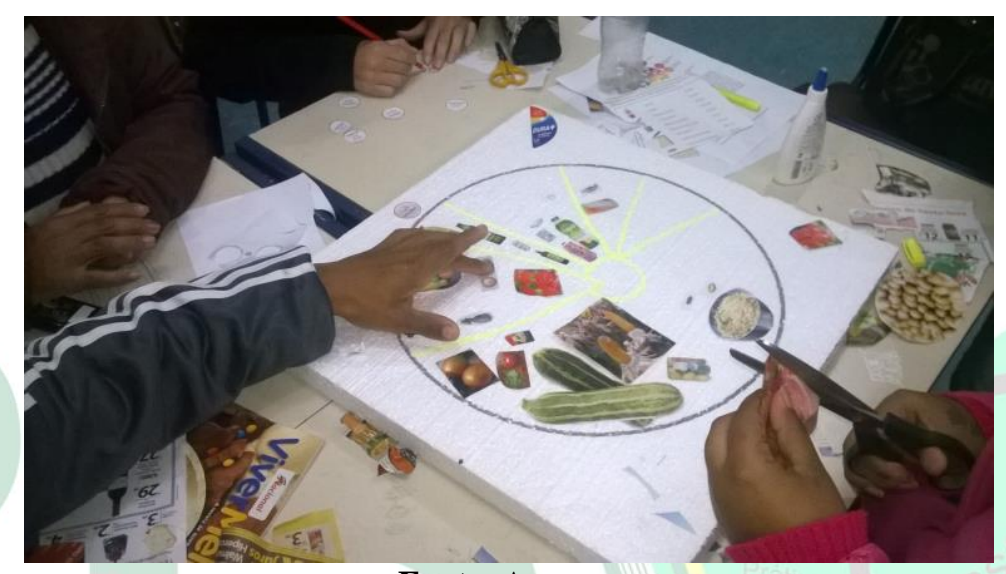

Fonte: Autoras.

Imagem 01- Alunos construindo a roda de alimentos.

$\mathrm{Na}$ terceira etapa, ainda referente ao momento pedagógico da organização do conhecimento, os participantes da oficina, assistiram a um vídeo ${ }^{3}$. Esta atividade proporcionou aos estudantes, fácil entendimento dos alimentos que devemos combinar no dia a dia para mantermos uma alimentação equilibrada e saudável.

Após, foi realizada a receita de um cupcake com casca de maçã. Durante sua produção, foram introduzidos conceitos de Ciências da Natureza relacionados a temática Alimentação. Nesta etapa da oficina, os participantes encontraram muita dificuldade em realizar os cálculos relacionados ao fornecimento de energia da receita produzida, em que muitos não demonstraram interesse no assunto, alegando que era complicado realizar as conversões de unidades de medida, mesmo com a utilização da calculadora. Apesar das dificuldades apresentadas, os estudantes conseguiram desenvolver o que foi solicitado.

Após o estudo dos conceitos de Ciências da Natureza relacionados à produção do cupcake, ocorreu a degustação da receita produzida. Pretendeu-se com a realização

${ }^{3}$ Disponível em:< https://www.youtube.com/watch?v=PtfyEYGQsf0>. Acesso 20 nov 2019. 
das atividades, uma maior conscientização por parte dos estudantes com respeito à ações sustentáveis que gerem alternativas mais saudáveis para o nosso planeta. Levando-os a compreender que comendo corretamente, também vive-se melhor.

No momento pedagógico da aplicação do conhecimento, realizado na última etapa, os estudantes com o auxílio de um software 4 para criação de infográficos, tiveram a oportunidade de ilustrar o que aprenderam durante a realização das oficinas. Esperavase que este tipo de representação visual gráfica, facilitasse a exposição dos conteúdos estudados, porém, os estudantes encontraram dificuldades em manusear esta ferramenta, sendo que a maior parte da turma não conseguiu utilizar o software e, somente um grupo obteve resultados. Então, foi utilizada uma segunda alternativa para os estudantes que não estavam conseguindo manusear o infográfico. Estes, fizeram uso do Power Point $^{\circledR}$ e, tiveram a oportunidade de ilustrar o que aprenderam durante a oficina. A Imagem 02 apresenta um dos infográficos elaborados pelos alunos:

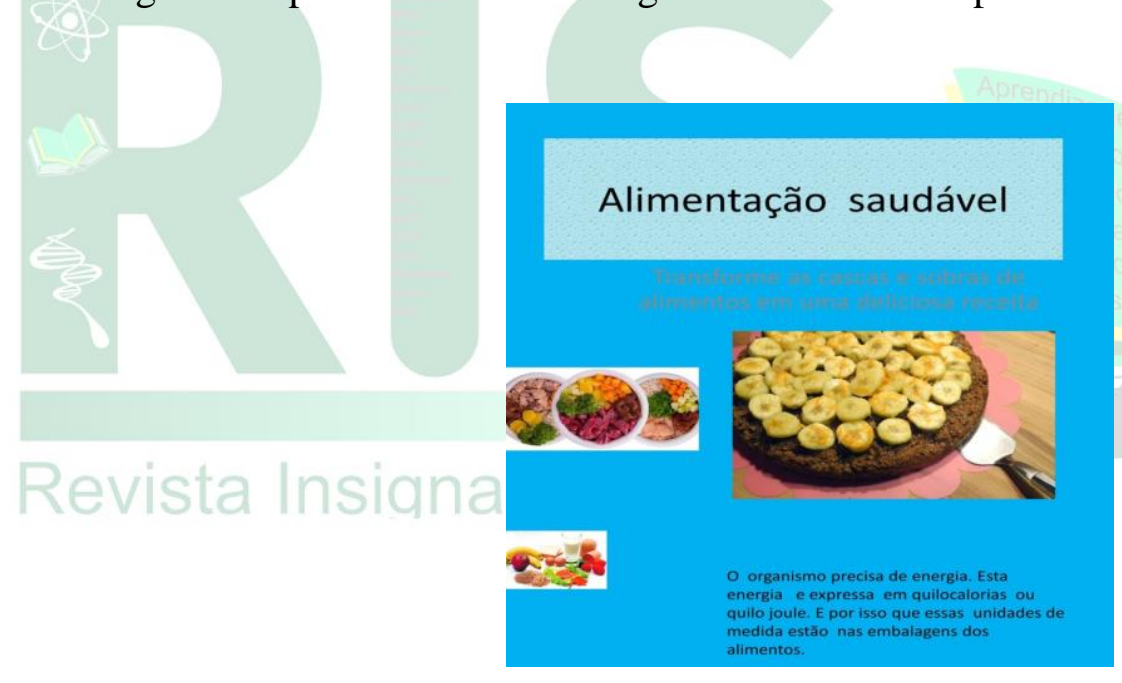

Imagem 02 - Infográfico elaborado por um dos grupos de participantes da pesquisa.

$\mathrm{Na}$ análise dos trabalhos elaborados na etapa da aplicação do conhecimento, pode-se perceber que os participantes da oficina relacionaram principalmente o conceito de energia ao tema Alimentação. Com relação ao conceito de energia, verificou-se também, que alguns grupos mencionavam a energia química fornecida pelos alimentos, outros, enfatizaram a propagação de energia térmica no preparo das refeições e, em algumas representações, houve menção à energia mecânica que deriva da conversão da energia fornecida pela ingestão dos alimentos. Também foi possível observar, que os

\footnotetext{
${ }^{4}$ https://piktochart.com/
} 
estudantes passaram a conceituar de forma correta as expressões: alimentação sustentável, alimentação saudável e alimentos orgânicos, o que demonstrou que o desenvolvimento da oficina proporcionou a construção de conhecimento referente à temática, visto que, no questionário inicial, esses conceitos ainda não estavam internalizados pelos estudantes. Os infográficos elaborados pela turma, foram expostos na escola, buscando apresentar o tema a toda comunidade escolar.

\section{CONSIDERAÇÕES FINAIS}

O desperdício dos alimentos é uma triste realidade que está presente em nosso cotidiano. Parte dos alimentos que normalmente vão parar no lixo, podem ser aproveitados de maneira integral, reduzindo este cenário. $\mathrm{Na}$ escola o professor pode orientar seus alunos na adoção de hábitos de vida, que visem produzir uma alimentação que não cause impactos ambientais. Esse relato de experiência buscou descrever a contribuição de uma oficina temática no processo de construção do conhecimento de estudantes do Ensino Médio, para compreender os conceitos de Ciências da Natureza relacionados à alimentação.

Após o desenvolvimento da oficina constatou- se o interesse dos estudantes de Educação Básica por atividades práticas sobre a temática. Apesar das dificuldades apresentadas pelos participantes com relação aos conceitos de Ciências da Natureza relacionados ao aproveitamento total dos alimentos, na última etapa da pesquisa, verificou-se que os conteúdos foram internalizados de forma significativa pela maioria dos estudantes, que conseguiram identificar a relação entre o alimento ingerido e a energia química, e outros conceitos como conservação de energia, energia e potência mecânica. Os estudantes também compreenderam a propagação de energia térmica no preparo de alimentos que necessitam ser aquecidos em fornos, fogões, micro-ondas etc. Esses resultados foram apresentados nos infográficos elaborados pela turma e, posteriormente expostos na escola. O Ensino de Ciências pode contribuir de forma significativa para o exercício da cidadania, sendo que o professor deve estar em busca de novas metodologias de ensino, atrelando a prática ao cotidiano do aluno. 


\section{REFERÊNCIAS}

AMARAL, A. de S.; ALMEIDA, A. S. Atividade interdisciplinar sobre Educação Alimentar aplicada no Colégio Estadual Dr. Orlando Leite em Vitória da Conquista - BA. Pacto pelo fortalecimento do Ensino Médio Bahia. Disponível em $<$ http://www.emdialogo.uff.br/content/atividade-interdisciplinar-sobre-educacaoalimentar-aplicada-no-colegio-estadual-dr-orlando> Acesso em 06 nov 2015.

BAUMGRATZ, C. E; et al. Reflexões sobre o papel da alimentação saudável no contexto escolar. Revista Insignare Scientia, v. 2, n. 2, 2019, p. 266- 276. Disponível em: <https://periodicos.uffs.edu.br/index.php/RIS/article/view/10995/7223>. Acesso 09 mar 2021.

BONATTO, A. et al. Interdisciplinaridade no Ambiente Escolar. In: SEMINÁRIO DE PESQUISA DA REGIÃO SUL, 9, 2012. Caxias do Sul. Disponível em < http://www.ucs.br/etc/conferencias/index.php/anpedsul/9anpedsul/paper/viewFile/2414/ 501> Acesso em 16 set 2015.

BRASIL. Ministério da Educação. Diretrizes Curriculares Nacionais do Ensino Médio. Brasília: MEC, 1998.

BRASIL. Ministério da Educação. Secretaria de Educação Média e Tecnológica. Parâmetros Curriculares Nacionais: Ensino Médio. Brasília: Ministério da Educação, 2002.

DELIZOICOV, D; ANGOTTI, J.A. Metodologia do ensino de ciências. São Paulo: Cortez, 1994.

FEISTEL, R. A. B. Contribuições da perspectiva freireana de educação para a interdisciplinaridade na formação inicial de professores de Ciências. 2012. Tese (Doutorado em Educação Científica e Tecnológica) - Universidade Federal de Santa Catarina, Florianópolis, 2012.

JAIME, P. C. et al. Um olhar sobre a agenda de alimentação e nutrição nos trinta anos do Sistema Único de Saúde. Ciência \& Saúde Coletiva, n. 23, 2018, p. 1829 - 1836.

LOCATELLI, A.; CRESTANI, E. R. M. F.; ROSA, C. T. W. da. Os três momentos pedagógicos e a interdisciplinaridade no Ensino de Ciências da Natureza: análise de um curso de formação continuada. Revista Insignare Scientia, v. 3, n. 1, 2020, p. 188 211. Disponível em < https://periodicos.uffs.edu.br/index.php/RIS/article/view/11137/7382>. Acesso em 09 $\operatorname{mar} 2021$.

MARCONDES, M. E. R. Org. Oficinas temáticas no ensino público visando a formação continuada de professores. São Paulo: GEPEQ, USP, 2006. 
MARCONDES, M. E. R. Proposições metodológicas para o ensino de química: oficinas temáticas para a aprendizagem da ciência e o desenvolvimento da cidadania. Em Extensão, Uberlândia, v. 7, 2008.

MONTEIRO, N. S. de O. Alimentação em tempos de crise - estudo de um caso prático. 2015 - Dissertação de Mestrado (Ciências do Consumo Alimentar). Universidade Aberta, 2015.

MORAES, M. C. A formação do educador a partir da complexidade e da transdisciplinaridade. Diálogo Educacional, v.7, n. 22, p. 13-38, 2007.

MORIN, E. Sete Saberes Necessários para uma Educação do Futuro. 2 ed. São Paulo: Cortez, 2000.

PINHEIRO, A. Reflexões sobre o processo histórico/ político de construção da lei orgânica de segurança alimentar e nutricional. Segurança Alimentar e Nutricional, 2008, n. 15, p. 1-15.

RODRIGUES, S., et al. A new food guide for the Portuguese population: development and technical considerations. Journal of Nutrition and Education Behavior, n. 38, 2006, p. 189-95.

VASCONCELOS, F. Combate a fome no Brasil: uma análise histórica de Vargas a Lula. Revista Nutri, n. 18, 2005, p. 439-457.

VYGOTSKY, L.S. A formação social da mente. 6. ed. SP: Martins Fontes, 2000. 